\title{
Diagnosing Topological Edge States via Entanglement Monogamy
}

\author{
K. Meichanetzidis, ${ }^{1, *}$ J. Eisert, ${ }^{2}$ M. Cirio, ${ }^{3}$ V. Lahtinen, ${ }^{2}$ and J. K. Pachos ${ }^{1}$ \\ ${ }^{1}$ School of Physics and Astronomy, University of Leeds, Leeds LS2 9JT, United Kingdom \\ ${ }^{2}$ Dahlem Center for Complex Quantum Systems, Freie Universität Berlin, 14195 Berlin, Germany \\ ${ }^{3}$ Interdisciplinary Theoretical Science Research Group (iTHES), RIKEN, Wako-shi, Saitama 351-0198, Japan
}

(Received 1 December 2015; published 30 March 2016)

\begin{abstract}
Topological phases of matter possess intricate correlation patterns typically probed by entanglement entropies or entanglement spectra. In this Letter, we propose an alternative approach to assessing topologically induced edge states in free and interacting fermionic systems. We do so by focussing on the fermionic covariance matrix. This matrix is often tractable either analytically or numerically, and it precisely captures the relevant correlations of the system. By invoking the concept of monogamy of entanglement, we show that highly entangled states supported across a system bipartition are largely disentangled from the rest of the system, thus, usually appearing as gapless edge states. We then define an entanglement qualifier that identifies the presence of topological edge states based purely on correlations present in the ground states. We demonstrate the versatility of this qualifier by applying it to various free and interacting fermionic topological systems.
\end{abstract}

DOI: 10.1103/PhysRevLett.116.130501

Consider a two-dimensional gapped system prepared in a pure state $\rho$ partitioned into region $A$ and its complement $B$. For large enough regions with smooth boundaries, the entanglement entropy corresponding to the reduced density matrix $\rho_{A}=\operatorname{tr}_{B}(\rho)$ is expected to take the form $S\left(\rho_{A}\right)=$ $(\alpha+\gamma)|\partial A|-\gamma+O\left(|\partial A|^{-\beta}\right)$, where $\alpha, \beta, \gamma \geq 0$ are constants and $\partial A$ denotes the boundary of $A$ [1]. The first term describes the area law contribution that is generally considered to be nonuniversal since $\alpha$ depends on system specific microscopics and can change adiabatically [2]. In contrast, the second term $\gamma$ is a universal constant called the topological entanglement entropy [3-5]. The numerical extraction of $\gamma$ has become a feasible numerical instrument to identify topologically ordered states in strongly correlated systems [6].

Topological phases of fermions, commonly referred to as topological insulators and superconductors, can have a band structure that is characterized by nontrivial topological indices [7] even if $\gamma=0$. A physical consequence of this is the appearance of edge states at their boundaries [8], that can be used as a means to identify topological phases theoretically $[9,10]$ or in the laboratory [11]. Edge states are eigenstates of the Hamiltonian that are exponentially localized at the boundary of the system and minimally coupled to the rest of the system. Because of the equivalence of the entanglement spectrum [12]-the spectrum $\left\{\epsilon_{j}\right\}$ of the virtual entanglement Hamiltonian $H_{A}$ defined by $\rho_{A}=e^{-H_{A}} / \operatorname{tr}\left(e^{-H_{A}}\right)$-with the physical energy

Published by the American Physical Society under the terms of the Creative Commons Attribution 3.0 License. Further distribution of this work must maintain attribution to the author(s) and the published article's title, journal citation, and DOI. spectrum [13], virtual entanglement edge states are also witnessed in the spectrum of $\rho_{A}$ in topological phases. The virtual edge states are also exponentially localized at the partition boundary $\partial A$, thus, being minimally correlated to the bulk states of $A$ but highly entangled to the complementary subsystem $B$. While both the energy and entanglement spectra can be adiabatically tuned, topology implies that the virtual edge states persist unless the bulk energy gap closes. Hence, topological phases cannot be adiabatically connected to a product state with $\alpha=0$ [2]. However, to use this entropic criterion to identify topological phases requires full diagonalization of the model, which is, in general, challenging, particularly in the presence of interactions.

Here, we take an entirely different approach to identify edge states and topological phases in both free and interacting fermionic systems. In contrast to entropic witnesses that address collective mode effects, we focus on two-point correlations. These are conceptually simpler objects, and at the same time ones that can be numerically much more easily obtained by, e.g., tensor network [14] or Monte Carlo methods $[15,16]$. We show that the high entanglement of individual virtual edge states is efficiently captured by the fermionic covariance matrix even in interacting models. Our argument is based on the extremality property of Gaussian fermionic states conjunct with the notion of the monogamy of entanglement $[17,18]$. In terms of entanglement entropy, we show how to single out the area law contributions of the virtual edge states from two-point correlations, without the need to study system-size scaling or needing to adiabatically tune the Hamiltonian. Finally, we demonstrate that this correlation based signature, unlike spectral signatures of topological phases, is robust against perturbations, disorder, and interactions. 
The covariance matrix.-First, we introduce the covariance matrix that facilitates our study of edge state correlations. A physical system that embodies $N$ fermionic modes with annihilation operators $f_{1}, \ldots, f_{N}$ can always be associated with $2 N$ Hermitian Majorana fermions $\gamma_{1}, \ldots, \gamma_{2 N}$, by $f_{j}=\left(\gamma_{2 j-1}+i \gamma_{2 j}\right) / \sqrt{2}$, for $j=1, \ldots, N$. The second moments of such Majorana fermions of an arbitrary fermionic density matrix $\rho$ can be collected in the covariance matrix $\Gamma[13,19-21]$, whose elements are the two-point correlations

$$
\Gamma_{j, k}=i \operatorname{tr}\left(\rho\left[\gamma_{j}, \gamma_{k}\right]\right) .
$$

This is a real $2 N \times 2 N$ matrix which is well defined for arbitrary fermionic states, including ground states of superconducting or interacting models, and allows us to treat them all on an equal footing. Since it satisfies $\Gamma=-\Gamma^{T}$ and $\Gamma^{T} \Gamma \leq \mathbb{1}$, it has eigenvalues $\left\{\mu_{j}\right\} \in[-1,1]$ coming in positive and negative pairs. For an arbitrary bipartition of the system in regions $A$ and $B$, the covariance matrix can be written as

$$
\Gamma=\left[\begin{array}{cc}
\Gamma_{A} & \Gamma_{A B} \\
-\Gamma_{A B}^{T} & \Gamma_{B}
\end{array}\right],
$$

with $\Gamma_{A(B)}$ reflecting the second moments of the reduced state $\rho_{A(B)}$ and $\Gamma_{A B}$ capturing correlations between $A$ and $B$.

For free fermionic systems all ground states are Gaussian states and, as such, are completely defined by the covariance matrix $\Gamma$. The positive eigenvalues $\left\{\mu_{j}^{A}\right\}$ of the covariance matrix $\Gamma_{A}$ are in one-to-one correspondence with the entanglement spectrum $\left\{\epsilon_{j}\right\}$ through the relation $\mu_{j}^{A}=$ $\left(1-e^{\epsilon_{j}}\right) /\left(1+e^{\epsilon_{j}}\right)$. For pure Gaussian states, one finds that the singular values $\xi_{j}^{A B}$ of $\Gamma_{A B}$ and the eigenvalues of $\Gamma_{A}$ satisfy $\left(\mu_{j}^{A}\right)^{2}+\left(\xi_{j}^{A B}\right)^{2}=1$. The entanglement entropy can be evaluated by summing over the contributions from each mode $S\left(\rho_{A}\right)=-\frac{1}{2} \sum_{j=1}^{2 N}\left(1+\mu_{j}^{A} / 2\right) \log \left(1+\mu_{j}^{A} / 2\right)$. Thus, the modes with $\mu_{j}^{A}=0$ (always coming in pairs) are uncorrelated with the rest of $A$ and are maximally entangled with modes in $B$ witnessed by $\xi_{j}^{A B}=1$. They correspond to the virtual edge states that translate to maximally entangled modes in the entanglement spectrum of topological free fermion systems, which contribute a maximal entropy of $\frac{1}{2} \log (2)$ per mode [13].

Next, we turn to studying the properties of edge states of interacting fermions by considering the highly correlated modes with $\xi_{j}^{A B} \approx 1$. To relate these states to the virtual edge states, we employ the monogamy of entanglement. This is a powerful general principle that allows us to detect edge states from the eigenvalues of the covariance matrix $\Gamma$.

Entanglement monogamy.-Monogamy of entanglement states that no mode in $A$ maximally entangled with a mode in $B$ can be entangled with any other mode in $A$ or $B$ $[17,18]$. In the language of covariance matrices, singular values $\xi_{j}^{A B}=1$ of $\Gamma_{A B}$ imply an eigenvalue $\mu_{j}^{A}=0$ (note that the converse is not necessarily true). Thus, such uncorrelated modes within $A$ must be decoupled from the bulk states and appear as exponentially localized states at the boundary of $A$.

To make this property more general and, thus, applicable to realistic systems, we consider the concept of monogamy in the presence of high entanglement between $A$ and $B$ that is not necessarily maximal. We start by discussing a two-mode subsystem. Let $\Xi$ be any principal $4 \times 4$ submatrix of the covariance matrix of an arbitrary bipartite fermionic state with reduced state $\sigma$. This matrix can be brought into the form

$$
\Xi=\left[\begin{array}{cccc}
0 & a & 0 & b \\
-a & 0 & c & 0 \\
0 & -c & 0 & d \\
-b & 0 & -d & 0
\end{array}\right]=\left[\begin{array}{cc}
\Xi_{A} & \Xi_{A B} \\
-\Xi_{A B}^{T} & \Xi_{B}
\end{array}\right] .
$$

This is a consequence of the real special orthogonal singular value decomposition, applied to both local modes individually. This covariance matrix corresponds to a pure maximally entangled state exactly if $|b|=|c|=1$, as only then $\Xi^{T} \Xi=\mathbb{1}$. Invoking the Jordan-Wigner transformation, the state $\sigma$ can be written as $\sigma=(1-\epsilon) \omega+\epsilon \eta$, where $\omega$ is a maximally entangled state of minimum dimension, $\min (|b|,|c|)>1-\epsilon$ is the smallest singular value of $\Xi_{A B}$, and $\eta$ an orthogonal residual state. In other words, we can argue about the weight of a maximally entangled state by considering only the covariance matrix rather than the full state $\sigma$ of the system. In particular, if $\min (|b|,|c|)>1-\epsilon$, then

$$
\|\sigma-\omega\|_{1} \leq 2 \epsilon,
$$

with the trace distance defined as $\|A\|_{1}=\operatorname{tr}(|A|)$ for operators $A$. Hence, by considering the eigenvalues of $\Xi_{A B}$, we can deduce how close the state $\sigma$ of the system is to a maximally entangled state $\omega$. The same argument applies to an arbitrary number of modes. If $2 k$ singular values of $\Xi_{A B}$ are larger than $1-\epsilon$, then one can identify a subspace embodying $k$ pairs of fermionic modes that are in trace distance closer than $2 \epsilon$ to $k$ maximally entangled pairs.

The almost maximally entangled modes are largely disentangled from the remaining fermionic modes, as dictated by the monogamy of entanglement $[17,18]$. To make this notion more precise, let us first focus on the situation of the reduced state supported on modes $S_{1}$ and $S_{2}$ being in a close to maximally entangled state; this is meant in the sense that this reduced state can be written as $\sigma=(1-\epsilon) \omega+\epsilon \eta$ as above. Then, mode $S_{1}$ will be little entangled with any other individual mode of the system. In fact, the sum of all entanglements of formation $E_{F}(1: j)$ [22] between $S_{1}$ and any other mode $S_{j}$ is upper bounded by

$$
\sum_{j=2}^{N} E_{F}(1: j) \leq \log (2)\left[1-(1-\epsilon)^{2}\right],
$$

and, hence, is small if $\epsilon$ is close to zero. This is a consequence of the following facts. The entanglement of formation $E_{F}$ and the tangle $\tau[17,18]$ are related as $E_{F}^{2} \geq \tau$. Then, the 
entanglement of formation is an entanglement monotone, so that $E_{F}(1: 2) \geq 1-\epsilon$ holds true. Finally, we make use of the monogamy of entanglement inequality for the tangle as discussed in Ref. [18].

The close to maximally entangled pair on $S_{1}$ and $S_{2}$ is also monogamous and disentangled in a different sense. These pairs are, as a whole, minimally entangled with all other modes, in the sense that

$$
E_{F}(1,2: 3, \ldots, N) \leq 2 \log (2) \epsilon .
$$

This bound is derived by using the convexity of the entanglement of formation and noting that the maximally entangled (Dirac fermion) pair takes the value $\log (2)$ in the chosen convention. Again, it is straightforward to generalize this argument to the case where $2 k$ singular values are larger than $1-\epsilon$. Then, the respective $k$ modes are, at most, $2 k \log (2) \epsilon$ entangled with the modes forming the complement of the system.

This result is general and applies to both free and interacting fermionic systems alike. It states that, due to their maximal correlations across $\partial A$, virtual edge modes appear as largely disentangled from the rest of the system. In 1D systems, this decoupling dictates that the edge states appear as zero modes in the entanglement spectrum. In 2D or 3D systems, they appear as gapless states freely propagating at $\partial A$.

Entropic lower bound.-The existence of virtual edge states implies a lower bound for the entanglement entropy [23]. In terms of the correlation part of the covariance matrix [24],

$$
S\left(\rho_{A}\right) \geq \frac{1}{2}\left\|\Gamma_{A B}\right\|_{2}^{2} \log (2),
$$

where the 2-norm is defined as $\|A\|_{2}^{2}=\operatorname{tr}\left(A^{2}\right)$, the sum of the squared singular values. Note that (7) is general, holding for free and interacting systems alike. For its interpretation, note that the contributions from the bulk states to the entanglement entropy can be adiabatically removed. But the fundamental properties of virtual edge states are resilient against any adiabatic evolution of the corresponding physical Hamiltonian [7]. Since topological phases are characterized by $\left\|\Gamma_{A B}\right\|_{2}^{2} \neq 0$, then the lower bound (7) becomes nontrivial, dictating that the area law coefficient $\alpha$ can never be made zero.

Entanglement qualifier.-We have shown above that nearly maximally entangled modes of the system are witnessed by singular values $\xi_{j}^{A B} \approx 1$ of $\Gamma_{A B}$. In analogy with the entanglement gap [25], these are separated by a covariance gap from the $\xi_{j}^{A B}$ corresponding to nonuniversal bulk states. This gives an efficient diagnostic tool to probe the topological character of the system. To count the number of such modes in a way robust to imperfections and finite system sizes, we define the entanglement qualifier $\mathcal{S}_{q}$ for some positive integer $q$ as

$$
\mathcal{S}_{q}=\operatorname{Tr}\left(\Gamma_{A B}^{\dagger} \Gamma_{A B}\right)^{q} .
$$

In the limit $q \rightarrow \infty$, this quantity converges to $M$-the number of maximally entangled modes in units of
Majorana modes (a Dirac mode counts as two Majorana modes) - and, thus, detects the existence of physical edge states from the ground state. In the presence of a covariance gap, $\mathcal{S}_{q}$ gives the degeneracy of these states for large but infinite values of $q$.

To demonstrate that the qualifier $\mathcal{S}_{q}$ identifies the presence of edge states and the size of their akin Hilbert space, we first apply it to Kitaev's honeycomb lattice model (2D topological superconductor) [26] and Haldane's model (2D Chern insulator) [27], in the presence and absence of disorder. In these free models, $\Gamma$ contains all the information about the ground state, while for interacting systems, this is not the case. Nevertheless, we show, in the context of the 1D SuSchrieffer-Heeger (SSH) model [28], with interactions that, even if the spectral properties fail to identify virtual edge states, due to monogamy, the covariance matrix successfully identifies their presence even for strong interactions.

Free fermionic models.-Kitaev's model is equivalent to free Majorana fermions $\gamma_{i}$ on a honeycomb lattice coupled to a $\mathbb{Z}_{2}$ gauge field. The time-reversal symmetry broken variant is defined by the Hamiltonian [24]

$$
H=\frac{i}{2} \sum_{\langle i, j\rangle} J_{i, j} u_{i, j} \gamma_{i} \gamma_{j}+\frac{i}{2} K \sum_{\langle i, j\rangle\rangle} u_{i, k} u_{k, j} \gamma_{i} \gamma_{j},
$$

where $J_{i, j}$ and $K$ are the nearest-neighbor and next-nearestneighbor hopping amplitudes. The link variables $u_{i, j}= \pm 1$ are conserved quantities that define different vortex sectors that support various topological phases characterized by different Chern numbers $\nu[29,30]$. We consider three different regimes with a distinct edge spectrum: (i) the $\nu=0$ Abelian phase with no edge states, (ii) the $\nu=1$ chiral non-Abelian phase with a single Majorana edge state, and (iii) the $\nu=2$ chiral Abelian phase in the full-vortex sector with two Majorana edge states (see [24] for the parameter regimes). Despite their different Chern numbers, the topological entanglement entropy takes the same value of $\gamma=\log (2)$ for all of these cases and is, thus, unable to distinguish between them [31]. The full spectrum of eigenstates is readily obtained by exact diagonalization. Constructing the full covariance matrix [24] and evaluating the qualifier $\mathcal{S}_{q}$ for each of the cases (i), (ii), and (iii) for a two component boundary $\partial A$, we find that it quickly converges to the values $M=0, M=2$, and $M=4$, respectively, as shown in Fig. 1 (Left). This is in exact agreement with the Chern number of each phase [29]. Similar analysis is carried out for the Haldane model for complex fermions $f_{i}$,

$$
H=\sum_{\langle i, j\rangle} t_{1} f_{i}^{\dagger} f_{j}+\sum_{\langle i, j\rangle} t_{2} e^{i \phi} f_{i}^{\dagger} f_{j}+\text { H.c., }
$$

where $t_{1}, t_{2}>0$, and $\phi \in[-\pi, \pi]$. When this model is tuned to the Chern insulator phase characterized by $\nu=1$ [24], Fig. 1 (left) shows the qualifier $\mathcal{S}_{q}$ converging to $M=4$ consistent with the edge spectrum of a single Dirac fermion per edge. The qualifier $\mathcal{S}_{q}$ also detects topological edge states in the presence of local random disorder in the 

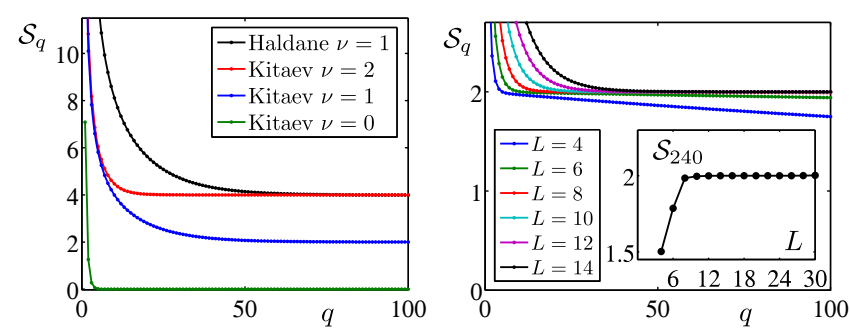

FIG. 1. (Left) Convergence of qualifier $\mathcal{S}_{q}$ for the Kitaev and Haldane models with a two-component boundary $\partial A$. For Kitaev's model, we find $\mathcal{S}_{q} \rightarrow 2 \nu$ consistent with each phase supporting $\nu$ Majorana edge states per boundary. For the Haldane model, we have $\mathcal{S}_{q} \rightarrow 4 \nu$ as a Dirac mode corresponds to two Majorana modes. (Right) System size $L=L_{x}=L_{y}$ dependence of the qualifier $\mathcal{S}_{q}$ for Kitaev's model with $\nu=1$, in the presence of disorder of magnitude $\Delta=0.5$. The data is averaged over 50 disorder realizations. The model parameters are given in Ref. [24].

Hamiltonian couplings. Indeed, Fig. 2 (right) shows that, in the disordered Kitaev model, the various topological phases are accurately identified with distinct quantized values of $\mathcal{S}_{q}$. We observe that disorder leaves the highly entangled states largely unaffected, mainly reducing, slightly, the singular values of $\Gamma_{A B}$ from the exact values 1 . Hence, a large but finite value of $q$ is appropriate for robustly identifying the topological phases.

In the idealized case of exactly maximal entangled virtual edge modes, one can take arbitrarily large values of $q$. Nevertheless, this is not always the case for finite-size systems or with the introduction of disorder, as shown Fig. 1 (right). In finite-size systems, the edge states can hybridize leading to smaller entanglement between them and, hence, a smaller lower bound. Moreover, the entanglement spectrum can exhibit even-odd effects in $|\partial A|$ that can wash out the lower bound completely [32]. Nevertheless, due to the exponential localization of the edge states, the lower bound can still be recovered in all cases via system-size scaling. Even-odd effects should vanish polynomially in $|\partial A|$, while decoupling of edge states occurs exponentially in the distance between boundary components. In the above examples, optimal choices of parameters are to take even cut length and a square system.

Interacting fermion model.-Now, we turn to the case of interacting fermions. We consider the 1D SSH model [28] of spinful fermions on a chain of length $L$ with staggered hopping and on-site interactions of strength $U$,

$$
\begin{aligned}
H= & \sum_{s} \sum_{i=1}^{L}-\left[t+\delta t(-1)^{i}\right] f_{i}^{s \dagger} f_{i+1}^{s}+\text { H.c. } \\
& +\frac{U}{2} \sum_{i=1}^{L}\left(n_{\uparrow, i}+n_{\downarrow, i}-1\right)^{2} .
\end{aligned}
$$

For periodic boundary conditions, the ground state is unique, while for open boundary conditions and for $U=0$, edges terminating on weak bonds host an edge mode per spin component. We half fill the system and restrict to the

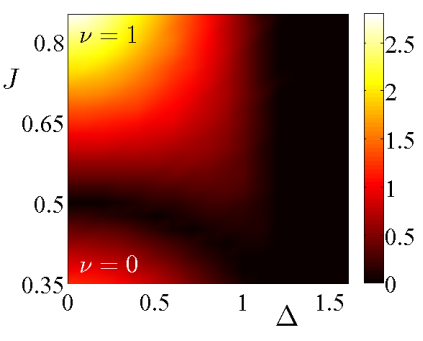

Energy gap

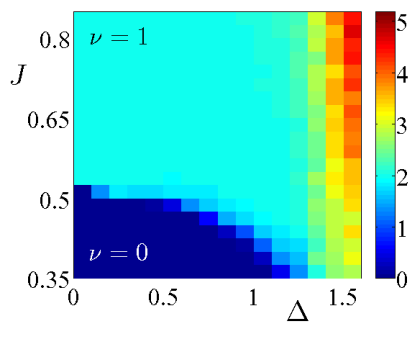

Qualifier $\mathcal{S}_{240}$
FIG. 2. (Left) Energy gap for the disordered vortex-free sector of Kitaev's model as a function of the coupling $J$ and the disorder $\Delta$. The topological phases with $\nu=0$ and $\nu=1$ are separated by a phase transition at $J_{c}=0.5$ for $\Delta=0$. The data is for $L_{x}=$ $L_{y}=30$ averaged over 50 disorder realizations of $J$ and $K$ [24]. (Right) The same phase diagram diagnosed by $\mathcal{S}_{q}$ showing extended regions where $\mathcal{S}_{240}=2|\nu|$ identifies the topological phases even for strong disorder. The nonquantized behavior for $\Delta>1$ identifies the thermal metal phase [30].

zero-spin sector which results in fourfold ground state degeneracy. Interactions linearly lift the degeneracy by hybridizing the spinful edge states, resulting in a ground state with twofold degeneracy [32], as shown in Fig. 3 (left).

Let us consider the system in terms of correlations. When $U=0$, the model is one of free fermions and the covariance matrix $\Gamma$ contains all the information about the ground state. Partitioning a periodic system in half with a twocomponent boundary, the qualifier $\mathcal{S}_{q}$ converges for large $q$ to $M=4$, consistent with two Dirac fermion edge modes per strong bond cut by $\partial A$ [24]. For $U>0$, the covariance matrix can still be readily evaluated from the eigenstates obtained via exact diagonalization, though in contrast to the free case, it does not contain all the information of the ground state. As shown in Fig. 3 (right), while the
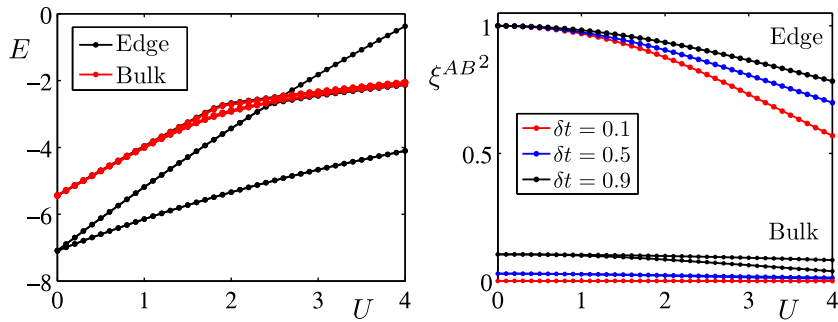

FIG. 3. (Left) Spectrum of the twelve lowest many-body states of the SSH model with interactions $U$ for $L=6$ and open boundaries terminating on weak bonds, $\delta t=0.75$. The two black lines are twofold degenerate. The ground state degeneracy reduces from fourfold $(U=0)$ to twofold $(U>0)$. (Right) The fourfold degenerate squared singular values $\xi_{j}^{A B 2}$ of $\Gamma_{A B}$ with periodic boundary conditions and equipartition (see Fig. 2 in Ref. [24]). While the degeneracy in the energy spectrum is lifted linearly with $U$ all the $M$ maximally entangled modes remain highly entangled and well separated from bulk entangled states by the covariance gap. Increasing $\delta t$ increases the entanglement of the edge states due to the decrease of the correlation length (equivalent to increasing the system size). 
degeneracy of the energy is lifted, all four virtual Majorana modes that are highly entangled across $\partial A$ behave identically in the covariance spectrum, a behavior not captured by the entanglement entropy [32]. They remain at high entanglement and are separated from the bulk states by the covariance gap. This also holds in the extreme case, where $U$ is large enough to cause the energies of the edge states to cross the bulk energies. This behavior of the qualifier $\mathcal{S}_{q}$ is consistent with the topological character of the system remaining unchanged. Indeed, there are no topological phase transitions and the winding number $[33,34]$ remains the same when $U$ is introduced [24]. In other words, interactions only change, adiabatically, the edge spectrum and the edge modes remain well defined. Thus, the covariance matrix can faithfully detect edge states and, thus, also identify topological phases in the presence of interactions, where $\Gamma$ no longer fully characterizes the ground state nor is in one-to-one correspondence with the entanglement spectrum.

Conclusions.-Here, we employed the covariance matrix to characterize topological phases in free and interacting fermion systems. We have shown that, due to the monogamy of entanglement $[17,18]$, the number of highly entangled modes probes the number of topologically induced edge states, regardless of their energy. Hence, the covariance matrix, similar to the Green's function [9], provides complementary information to the entanglement spectrum $[10,12]$. We demonstrated that the high entanglement of the virtual edge states, unlike their spectrum, is a robust characteristic under perturbations that leave the topological phase unchanged, such as disorder or interactions. This gives a systematic and unambiguous way to study edge states and, thus, the topological character of fermionic systems in theoretical and numerical investigations as well as in experiments.

V. L. acknowledges the support by Dahlem Research School POINT Fellowship program, K. M. by the EPSRC Grand No. EP/I038683/1, J. E. by the ERC (TAQ), and the EU (AQUS, RAQUEL, SIQS). We would like to thank I. Klich, G. Palumbo, Z. Papic, and C. Self for inspiring discussions.

*mmkm@leeds.ac.uk

[1] J. Eisert, M. Cramer, and M. B. Plenio, Rev. Mod. Phys. 82, 277 (2010).

[2] J. C. Budich, J. Eisert, and E. J. Bergholtz, Phys. Rev. B 89, 195120 (2014).

[3] A. Hamma, R. Ionicioiu, and P. Zanardi, Phys. Rev. A 71, 022315 (2005).

[4] A. Kitaev and J. Preskill, Phys. Rev. Lett. 96, 110404 (2006).

[5] M. Levin and X.-G. Wen, Phys. Rev. Lett. 96, 110405 (2006).

[6] H.-C. Jiang, Z. Wang, and L. Balents, Nat. Phys. 8, 902 (2012).
[7] A. P. Schnyder, S. Ryu, A. Furusaki, and A. W. W. Ludwig, Phys. Rev. B 78, 195125 (2008); A. Y. Kitaev, AIP Conf. Proc. 1134, 22 (2009).

[8] X.-L. Qi, Y.-S. Wu, and S.-C. Zhang, Phys. Rev. B 74, 045125 (2006).

[9] Z. Wang, X.-L. Qi, and S.-C. Zhang, Phys. Rev. Lett. 105, 256803 (2010).

[10] L. Fidkowski and A. Kitaev, Phys. Rev. B 83, 075103 (2011).

[11] M. Konig, S. Wiedmann, C. Brune, A. Roth, H. Buhmann, L. W. Molenkamp, X.-L. Qi, and S.-C. ZhangM. Konig, S. Wiedmann, C. Brune, A. Roth, H. Buhmann, L. W. Molenkamp, X.-L. Qi, and S.-C. Zhang, Science 318, 766 (2007).

[12] H. Li and F. D. M. Haldane, Phys. Rev. Lett. 101, 010504 (2008).

[13] L. Fidkowski, Phys. Rev. Lett. 104, 130502 (2010).

[14] P. Corboz, R. Orus, B. Bauer, and G. Vidal, Phys. Rev. B 81, 165104 (2010); P. Corboz, G. Evenbly, F. Verstraete, and G. Vidal, Phys. Rev. A 81, 010303(R) (2010); T. Barthel, C. Pineda, and J. Eisert, Phys. Rev. A 80, 042333 (2009).

[15] P. Broecker and S. Trebst, J. Stat. Mech. (2014) P08015.

[16] T. Grover, Phys. Rev. Lett. 111, 130402 (2013).

[17] V. Coffman, J. Kundu, and W. K. Wootters, Phys. Rev. A 61, 052306 (2000).

[18] T. J. Osborne and F. Verstraete, Phys. Rev. Lett. 96, 220503 (2006).

[19] I. Peschel, J. Phys. A 36, L205 (2003); I. Peschel and V. Eisler, J. Phys. A 42, 504003 (2009).

[20] S. Bravyi, Quantum Inf. Comput. 5, 216 (2005); D. P. DiVincenzo and B. M. Terhal, Found. Phys. 35, 1967 (2005).

[21] M. Cramer, J. Eisert, and M. B. Plenio, Phys. Rev. Lett. 98, 220603 (2007); H. Bernigau, M. J. Kastoryano, and J. Eisert, J. Stat. Mech. (2015) P02008.

[22] C. H. Bennett, H. J. Bernstein, S. Popescu, and B. Schumacher, Phys. Rev. A 53, 2046 (1996).

[23] I. Klich, J. Phys. A 39, L85 (2006).

[24] See Supplemental Material at http://link.aps.org/ supplemental/10.1103/PhysRevLett.116.130501 for details of the analytical arguments and a further discussion of the physical models under consideration.

[25] R. Thomale, A. Sterdyniak, N. Regnault, and B. A. Bernevig, Phys. Rev. Lett. 104, 180502 (2010).

[26] A. Kitaev, Ann. Phys. (Amsterdam) 321, 2 (2006).

[27] F. D. M. Haldane, Phys. Rev. Lett. 61, 2015 (1988).

[28] W. P. Su, J. R. Schrieffer, and A. J. Heeger, Phys. Rev. Lett. 42, 1698 (1979).

[29] V. Lahtinen, A. W. W. Ludwig, J. K. Pachos, and S. Trebst, Phys. Rev. B 86, 075115 (2012); V. Lahtinen and J. K. Pachos, Phys. Rev. B 81, 245132 (2010); J. K. Pachos, Ann. Phys. (Amsterdam) 322, 1254 (2007).

[30] V. Lahtinen, A. W. W. Ludwig, and S. Trebst, Phys. Rev. B 89, 085121 (2014).

[31] H. Yao and X.-L. Qi, Phys. Rev. Lett. 105, 080501 (2010).

[32] D. Wang, S. Xu, Y. Wang, and C. Wu, Phys. Rev. B 91, 115118 (2015).

[33] J. de Lisle, S. De, E. Alba, A. Bullivant, J. J. Garcia-Ripoll, V. Lahtinen, and J. K. Pachos, New J. Phys. 16, 083022 (2014).

[34] E. Alba, J. K. Pachos, and J. J. Garcia-Ripoll, New J. Phys. 18, 033022 (2016). 